values were normalized when expressed per kg LBM. Postprandial oxidation was reduced in elderly men relating to an increase in splanchnic extraction. These results suggested that a limited amino acid disposal to muscle in the elderly is a consequence of a higher splanchnic extraction that could, in turn, lead to a lower stimulation of muscle protein synthesis during feeding.

Plasma albumin concentration is a main factor in the control of lipoprotein metabolism: a kinetic study of two cases of analbuminemia. C Maugeais, K Ouguerram, P Maugeais, P Mahot, T Magot, M Krempf (Centre de recherches en nutrition humaine, hôpital Laënnec, 44035 Nantes cedex 01, France)

Hyperlipidaemia is commonly present in nephrotic syndrome. From previous studies, the physiopathology of these lipid disturbances could be related to a low albumin plasma level or to urinary loss of factors controlling the lipoprotein metabolism. Human analbuminemia is an inherited disease characterised by a low plasma albumin concentration and a dyslipidaemia but no proteinuria. This rare disease represents a good model for better understanding the mechanism of dyslipidaemia in nephrotic syndrome.

We studied the kinetic aspects of the apoB-containing lipoproteins metabolism of two sisters ( 26 and 30 years old) with analbuminemia using a constant infusion of leucine labeled with stable isotopes for $14 \mathrm{~h}$.

Compared to control subjects (male, 21-25 years old), very low density lipoprotein (VLDL)-apoB production was higher ( $41.5 \mathrm{vs} 24.8 \mathrm{mg} / \mathrm{kg} / \mathrm{day}$ ) as well as the production of intermediate density lipoprotein (IDL) and low density lipoprotein (LDL)-, apoB (30 vs 16 and 16 vs $10 \mathrm{mg} / \mathrm{kg} /$ day, respectively). The fractional catabolic rate of all apoB-containing lipoproteins was decreased $(0.30$ vs $0.48,0.28$ vs $0.62,0.011$ vs $0.022 \mathrm{~h}^{-1}$, respectively).

These abnormalities were similar to the kinetic disturbances previously reported for nephrotic syndrome. The results suggested that a low albumin plasma concentration is probably the main factor controlling the lipid abnormalities in this kidney disease.

\section{LIPID METABOLISM}

Hepatic steatosis in the goose: influence of genotype on lipid metabolism. E Fournier 1, G Guy 2, R Peresson 1, D Hermier 1 ( ${ }^{1}$ Inra, 37380 Nouzilly; ${ }^{2}$ InraArtiguères, 40280 Benquet, France)

Susceptibility to liver steatosis in the goose is at least partly under genetic control. The Landes goose (L) exhibits a typical fatty liver in response to overfeeding, whereas the Poland goose $(P)$ is partly resistant. Plasma and liver lipids were therefore analyzed in 14-week-old male geese weighing $5.2 \mathrm{~kg}$ at the start of the experimental period (16 $L$ and $13 P$ ). Plasma lipoproteins were isolated by density gradient ultracentrifugation [Hermier et al (1988) J Lipid Res 29, 893907] and their concentration and chemical composition were determined before and after 14 days of overfeeding with boiled maize. Liver composition was determined after the completion of the overfeeding period.

Before overfeeding, the plasma lipoprotein profile of the geese was typical of birds, having a low amount of very low density lipoproteins (VLDL), and a predominance of high density lipoproteins (HDL). In both breeds, VLDL were abnormally poor in triglycerides (TG $\approx 30 \%$ ), but HDL concen- 